\title{
Wenn Katze, dann schon in jungen Jahren
}

Eine allergische Sensibilisierung gegen Katzenallergene erhöht das Asthmarisiko. Doch ist immer noch nicht widerspruchsfrei geklärt, ob die Haltung einer Katze eher vor einer solchen Sensibilisierung schützt oder sie fördert. Eine prospektive Kohortenstudie liefert einen weiteren Beitrag zum Thema.

A ustralische Forscher dokumentierten im Jahr 1982 die Daten von Schülern im Alter zwischen 8 und 10 Jahren und verfolgten die „Allergiekarriere“ der Kinder bis ins Jahr 2002 weiter. Festgestellt wurde außerdem, ob die Studienteilnehmer im Alter von unter bzw. über 18 Jahren eine Katze zu Hause hielten.

Komplette Datensätze waren von 224 Teilnehmern verfügbar. 50 von ihnen hatten eine Katze lediglich im Alter von unter 18 Jahren gehalten, 14 ausschließlich im Alter über 18 Jahren und 70 in beiden Altersperioden. Verglichen mit den 90 Teilnehmern ohne Katzenhaltung war Katzenhaltung im Alter unter 18 Jahren mit einer Schutzwirkung gegenüber einer atopischen Sensibilisierung gegen Außenraumallergene, einer erhöhten bronchialen Hyperreagibilität, aktuellem Wheezing und Asthma verknüpft. Hatten sich die Studienteilnehmer die Katze dagegen erst im Alter von über 18 Jahren angeschafft, waren die Raten für Asthma und Atemwegshyperreagibilität leicht erhöht. Die Schutzwirkung eines engen Katzenkontakts in jungen Jahren blieb bis ins Erwachsenenalter bestehen, auch wenn aktuell keine Katze mehr gehalten wurde.

Erklärt werden könnte die beobachtete Schutzwirkung zum einen durch die Hygienehypothese: Die in Haushalten mit Haustieren erhöhten Endotoxinspiegel fördern die Verschiebung der immunologischen TH2-Antwort in Richtung einer nicht allergischen TH1-Antwort. Zum anderen könnte durch die erhöhte Exposition gegenüber den Katzenallergenen eine Immuntoleranz induziert worden sein. Das bedeutet u. a., dass sich eine TH2-Antwort eher in der Produktion von IgG4 als in der Produktion spezifischer IgE-Antikörper manifestiert. Da in dieser Studie aber weder die Allergen- noch die Endotoxinkonzentration in den Haushalten gemessen wurde, kann momentan keine klare Aussage über mögliche Mechanismen der Schutzwirkung gemacht werden.

Fazit: Katzenhaltung in einem Alter von unter 18 Jahren reduziert das Asthma- und Atopierisiko und schützt offensichtlich auch ohne weitergeführte Katzenhaltung vor einer allergischen Sensibilisierung.

de Meer $\mathrm{G}$ et al. Presence and timing of cat ownership by age 18 and the effect on atopy and asthma at age 28. J Allergy Clin Immunol 2004; 113: 433-8 